\title{
Experimental characterisation of holographic optical traps for microbubbles
}

\author{
Chris Fury ${ }^{\mathrm{a}, \mathrm{b}}$, Caroline Harfield ${ }^{\mathrm{a}, \mathrm{c}}$, Philip H. Jones ${ }^{\mathrm{b}}$, Eleanor Stride ${ }^{\mathrm{c}}$, Gianluca Memoli ${ }^{\mathrm{a},}{ }^{,}$ \\ ${ }^{a}$ Acoustics Group, National Physical Laboratory, Hampton Road, Teddington, TW11 8UQ, UK; \\ ${ }^{b}$ Dept. of Physics and Astronomy, University College London, Gower Street \\ London, WC1E 6BT, UK; ${ }^{\circ}$ Institute of Biomedical Engineering, Old Road Campus Research \\ Building, University of Oxford, Oxford OX3 7DQ, UK
}

\begin{abstract}
In this study microscopic gas bubbles (7-12 $\mu \mathrm{m}$ diameter) suspended in water were optically trapped in a custom-built microfluidic slide using holographically generated Laguerre-Gaussian ('doughnut') beam optical tweezers. The optical potential was then characterized as a function of bubble size, trapping laser power and trapping beam diameter (Laguerre-Gaussian beam mode) using the trap spring constant in the plane transverse to the beam propagation direction, obtained from the position fluctuations of the bubble in the trap measured by video microscopy and particle tracking. It was found that microbubbles were held at the equilibrium position of buoyant and optical forces at a distance from the focus of the beam that increased with laser power, and that optical trapping in this configuration was only possible within a specific range of trap and bubble parameters. Furthermore an optimum size of the doughnut beam to microbubble diameter which maximized the transverse spring constant was found. A ray optics model of the optical forces acting on microbubbles in a focused Laguerre-Gaussian beam was used in order to calculate the trap spring constants and equilibrium trapping position as a function of the different parameters, and highlight key physical behaviours.
\end{abstract}

Keywords: holographic trapping, microbubbles, low-index particles, characterization, ray optics model.

\section{INTRODUCTION}

Optical tweezers offer a powerful technique for the non-contact manipulation of colloidal materials, ranging from the nanoscopic [1] to the microscopic [2] regime. It was Ashkin et al. [3] who first demonstrated that a strongly focused laser beam could exert a significant force on dielectric particles with a higher refractive index than the medium in which they were suspended that was sufficient to confine them to the beam waist.

Gas bubbles on the order of $1-10 \mu \mathrm{m}$ in radius and stabilized by a coating of biological surfactant [4] have found use as contrast agents for ultrasound imaging and, more recently, have shown a clear potential as targeted drug delivery vectors [5]. The characterisation of the acoustical properties of these microbubbles is important in order to validate simulations and improve their design, thereby leading to safer, more clinically effective and cost-effective medical products. In order to perform this characterization at the single bubble level, a system has been constructed based upon simultaneous use of optical and acoustical trapping (NPL "sono-optical tweezers"), with the former providing the reference force calibration and a finer manipulation than the latter [6]. In the present study we characterize the optical potential as a function of laser beam (i.e. its power and diameter) and bubble size via comparison of the spring constant of the optical trap as a function of these parameters.

\subsection{Optical manipulation of microbubbles}

Low relative refractive index objects, such as microbubbles suspended in water, are more challenging to optically trap than high-index particles, as they are strongly repelled from the waist of a focused Gaussian laser beam by both scattering and gradient forces [7]. Microbubble trapping therefore requires alternative strategies. One method is to create a time-averaged optical potential by rapidly scanning a Gaussian laser beam around a microbubble at high frequency, thereby preventing it from diffusing away from the trap location [8].

*gianluca.memoli@npl.co.uk; phone 44(0)20 8943 6062; www.npl.co.uk/people/gianlucamemoli

Nanophotonics V, edited by David L. Andrews, Jean-Michel Nunzi, Andreas Ostendorf, Proc. of SPIE Vol. 9126, 91263L · @ 2014 SPIE · CCC code: 0277-786X/14/\$18 · doi: 10.1117/12.2055889 
Another possibility is to use a Laguerre-Gaussian laser beam [9][10] that, as a result of its phase structure, has an annular intensity profile that can confine a microbubble in the dark core. This solution has been applied to microbubbles to study their dynamics near a wall [11] or under acoustic excitation [12].

In both these trapping geometries the maximum transverse force that can be applied to the microbubble before it escapes from the trap has been determined [10][13]. Jones et al. [13] measured the trap spring constant as a function of laser power and microbubble size in the transverse plane by observing the capture dynamics of a microbubble as it was drawn into a scanning laser trap. The spring constant measures the curvature of the optical potential in the region of the bubble's equilibrium position, and is therefore more representative of the forces acting on the bubble.

In this work the optical potential of a microbubble confined in a Laguerre-Gaussian (LG or 'doughnut') beam trap was characterized by determining the transverse spring constant for this geometry. The spring constant was measured by video-microscopy of the Brownian fluctuations in the microbubble position, and subsequent particle tracking analysis. A ray optics model of optically trapped microbubbles in the LG beam trap is also presented, which highlighted key aspects of the physical behaviour of microbubbles in this form of optical trap.

\section{MATERIALS AND METHODS}

\subsection{Experimental set-up}

The optical set up was constructed upon a vibration isolating optical bench (Thorlabs). An upright microscope was designed using the base construction of the Thorlabs OTKB/M optical tweezers kit, but modified in order to incorporate additional instrumentation (see Figure 1).

The trapping laser was a single mode Nd:YAG laser (Laser Quantum, Ventus, maximum output power, $P=5 \mathrm{~W}$, wavelength, $\lambda=1064 \mathrm{~nm}$ ). The Laguerre-Gaussian (LG) laser mode required for optical trapping of bubbles was holographically generated via imprinting a helical phase, $\Phi=2 \pi L$ ( $L$ the topological charge) on to the beam via a phase only spatial light modulator (SLM), manufactured by Boulder Non-Linear Systems Inc (XY Series, 256x256 pixels). The SLM was modified by the manufacturer, so that it is possible to change its frame rate by changing the liquid crystal temperature: a fixed temperature of $30{ }^{\circ} \mathrm{C}$, giving a frame rate of $160 \mathrm{~Hz}$, was used in this work. Once shaped into the desired LG mode, the 1st order diffracted beam (containing up to $30 \%$ of the incident power) could be position controlled in the two transverse dimensions by applying a blazed phase grating across the beam, and the waist formed by focusing this beam could be moved in the third (axial) dimension by applying a quadratic (lensing) function ("defocus", in the following text). A two lens telescope in the $4 f$ configuration expanded the beam to a diameter equal to that of the back aperture of the objective lens and conjugated the SLM plane with the back aperture. Ensuring that the full lens aperture is used was important here, as otherwise the effective numerical aperture of the lens decreased [7]. Conjugation of the SLM and back aperture planes ensures that the beam remains centred on the aperture when the phase grating function was applied to move the trap position. The beam was spatially filtered by inserting an iris in the Fourier plane of the first $4 f$ lens, in order to ensure that there were no competing traps in the sample focal plane.

The trapping laser entered the microscope through a side port and was routed to the upper optics of the microscope, to be focused down into the sample plane. The focusing was provided by a high numerical aperture objective lens (Nikon, PLAN APO IR, 60x, 1.27NA, water immersion). The transmitted light was captured by an aspherical condenser (Thorlabs ACL2520) and directed toward a beam dump. A low pass filter before the beam dump allowed an LED illumination source to be coupled into the system, providing for illumination of the sample. A second low pass filter before the objective allowed this light to be detached from the trapping optical train and to be steered onto a CCD (Thorlabs DCU223M) for sample observation and video recordings with video frame rate up to 90fps.

The microbubble in the sample plane was contained in solution within a custom designed glass microchannel [W:0.43 mm, H: $0.33 \mathrm{~mm}, \mathrm{~L}: 2.5 \mathrm{~mm}$ ] that enables the bubbles to be manipulated simultaneously with optical and acoustical forces. Optical access to the fluidic channel was guaranteed by a $0.17 \mathrm{~mm}$ thick, polished flat surface above and below the trapping region. Acoustic forces, not used in this work, could be applied by an external PZT transducer, located at a corner of the structure (Figure 2a). This study used Expancel 461-WU20 (Akzo Nobel): thermosoftening plastic shell microspheres containing a low boiling point hydrocarbon, that upon heating causes the particle to permanently expand. Once expanded (in boiling water for one minute), they had the size distribution depicted in Figure $2 \mathrm{~b}$ (measured by optical microscopy), with a modal value of $8 \mu \mathrm{m}$. The inset in Figure $2 \mathrm{~b}$ shows the diameters of the selection of bubbles used in this study. 


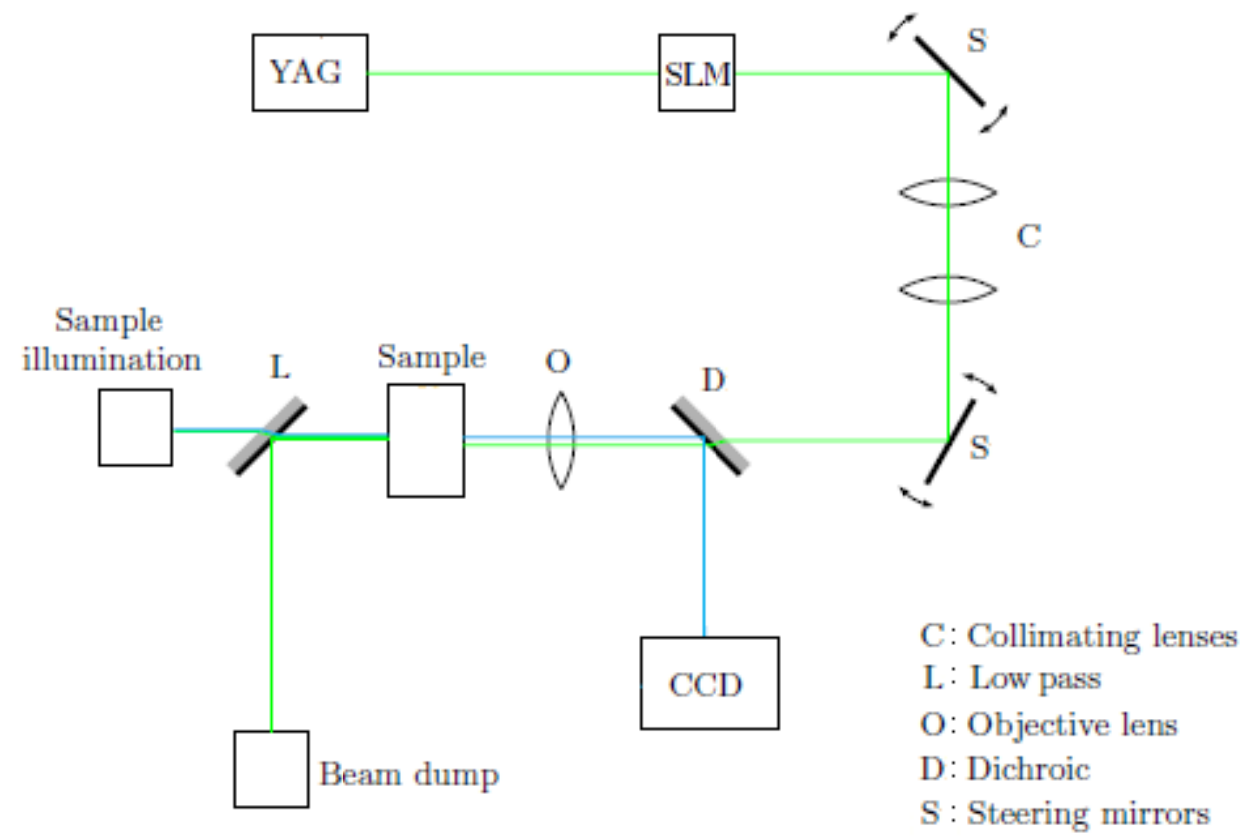

Figure 1. Ray path schematic of the optical set-up.
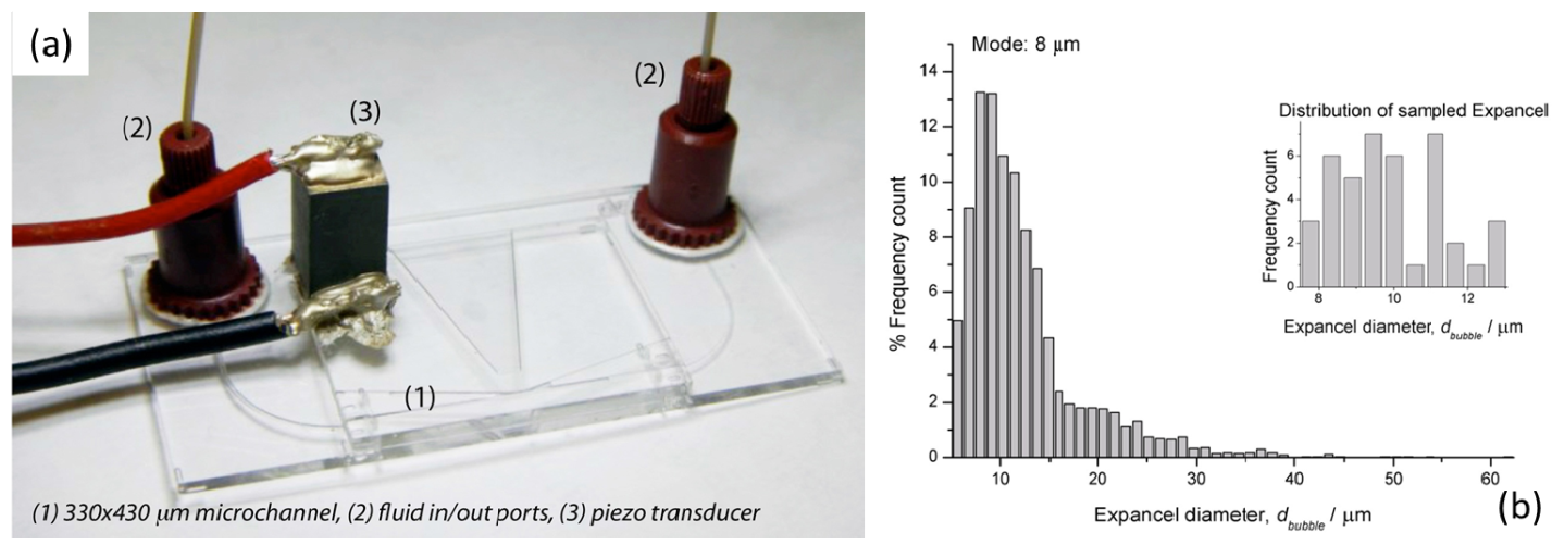

Figure 2. (a) Customised glass microchip used for the experiments. (b) size distribution of expanded Expancel 461-WU20, as determined by optical microscopy, The inset shows the size distribution of the bubbles used in this study.

\subsection{Data processing}

The trap stiffness in the transverse plane, $\kappa$, was determined through video-microscopy and particle tracking analysis [14]. The magnification of the imaging system was calibrated with a $200 \mu \mathrm{m}$ NPL reference stage graticule [15], enabling the trapped microbubble position fluctuations to be quantified. The frame rate of the CCD camera was limited by the size of the Region of Interest (ROI). To ensure the maximum rate is achieved, thus reducing overall acquisition time and video size, a cropping window was selected about the trapped bubble, whilst allowing for $\sim 20$ pixels of movement on each side (significantly greater than the extent of the position fluctuations). This achieved frame rates of 70-90 fps, depending on the bubble diameter. As a compromise between storage limits and the need for statistically relevant data, video recordings run until 4000 frames were captured and then saved for post processing. Video processing was performed in Matlab (Mathworks, 2013) using custom tracking software that was capable of determining the centroid of the image of the bubble and its diameter to sub-pixel accuracy. 
Assuming a quadratic shape for the trapping potential, it was possible to characterize the restoring force by monitoring the Brownian motion of each trapped bubble. The histograms of the bubble position fluctuations along a single dimension had a Gaussian profile, as shown in Figure 3, whose variance $\left\langle\mathrm{x}^{2}\right\rangle$ could be related to the spring constant via equipartition of energy as [16]:

$$
\kappa=\frac{k_{B} T}{\left\langle x^{2}\right\rangle}
$$

where $k_{B} T$ is the thermal energy.

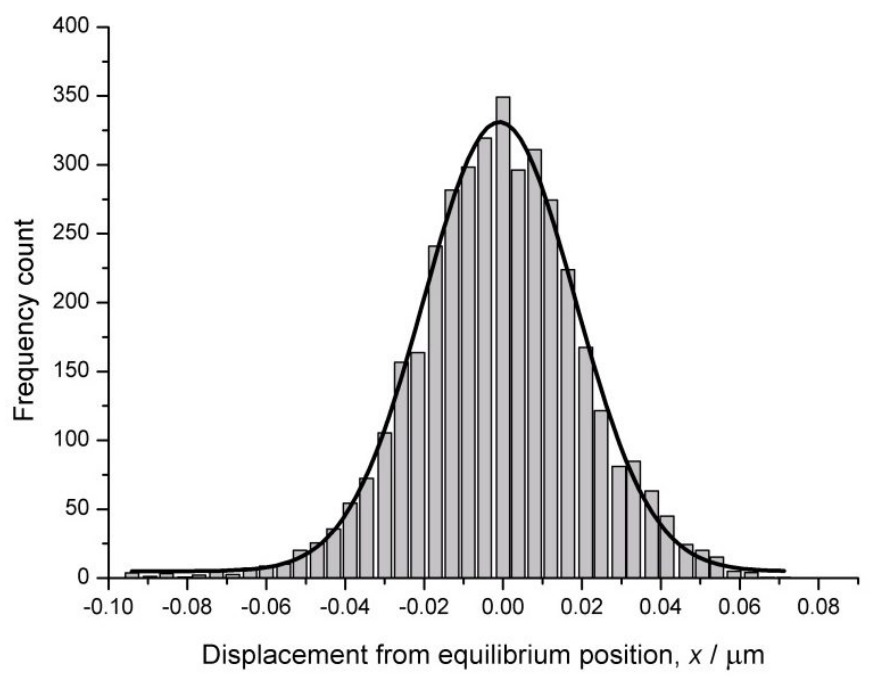

Figure 3. 1D histogram, with 40 bins, of bubble position fluctuations within an optical potential well. The fit describes a Gaussian profile of the histogram.

\section{MODELLING}

In order to interpret the behaviour of a microbubble in the LG beam trap a ray-optics model of the optical forces acting on the microbubble was derived. Since the size of the bubbles used in this study are comparable with the wavelength of the trapping laser, the accuracy of this model in terms of providing quantitative estimates of the trapping forces was somewhat limited. It should, however, provide a good qualitative description of the experimentally observed behavior.

\subsection{Ray Optics Model}

The model follows the derivation of Ashkin [7] to find the trapping efficiency, $Q$, of a LG beam optical trap for a low relative-refractive index microbubble. The trapping efficiency is a dimensionless parameter that characterizes the fraction of optical momentum transferred from the beam to the trapped object. As described in Figure 4, a ray initially parallel to the optical axis is incident on the objective at distance $r$ from the axis, with the objective lens of size $r_{\max }$. The ray is refracted towards the focus of the objective, $\mathrm{F}$, forming an angle $\varphi$ with the propagation direction, and in its path it encounters the bubble (in point $\mathrm{V}$ ), whose centre $\mathrm{O}$ is also the origin of the reference frame. The displacements $\mathrm{S} 1$ and S2 describe the position of the bubble relative to the focus, respectively in the radial and axial directions i.e. the focus $\mathrm{F}$ is positioned at $(0,-\mathrm{S} 1,-\mathrm{S} 2)$ in the reference frame centred in $\mathrm{O}$, as shown in Figure 4. The ray transfers a fraction of momentum $q_{\mathrm{s}}$ due to scattering (partial reflection) at the bubble surface in the direction $\hat{\mathbf{s}}$, parallel to the direction of propagation of the beam, and a fraction $q_{\mathrm{g}}$ due to refraction in the perpendicular direction, $\hat{\mathrm{g}}$. The net fractional momentum transfers (trapping efficiencies) due to scattering, $Q_{\mathrm{s}}$, and refraction, $Q_{\mathrm{g}}$, that produce the scattering and gradient forces are the intensity-weighted sum of all rays incident on the bubble from across the aperture. The trapping efficiencies were calculated as a function of microbubble position in order to find the force acting on the microbubble for a range of parameters of microbubble size, LG beam mode index (topological charge) and beam power. 


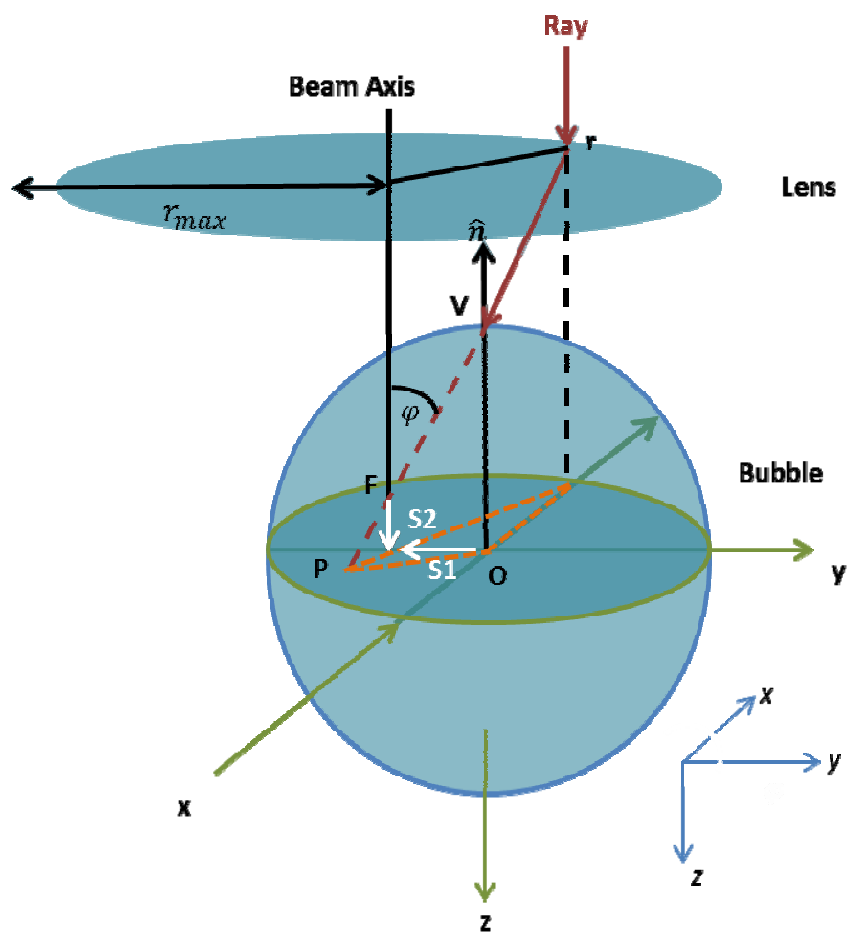

Figure 4. Trap geometry with the centre of the bubble located at S1 from the focus in the radial direction and a distance S2 in the $+\hat{z}$ direction.

\subsection{Trapping position for $L G$ modes}

The model was used to predict the equilibrium trapping position and the trap stiffness for a given set of input parameters (i.e. bubble radius, objective characteristics, working distance, LG beam mode, laser power) as follows:

1. Add a LG irradiance profile to the beam. The intensity weighting for each ray was adapted to the irradiance of a laser beam with a Laguerre-Gaussian (LG) profile overfilling the objective lens. Such that each ray carries a fraction $d P$ of the beam power:

$$
d P=\frac{2 \mathrm{P}_{0}}{\pi r_{\max }^{2}}\left(\frac{p !}{(L+p) !}\right)(-1)^{2 p} e^{-\left(\frac{2 r^{2}}{\left(r_{\max }^{2}\right)}\right)}\left(\frac{2 r^{2}}{r_{\max }^{2}}\right)^{|L|}\left(\Lambda_{p}^{L}\left(\frac{2 r^{2}}{r_{\max }^{2}}\right)\right)^{2} \times \sin (\alpha) r d r d \beta
$$

where $\Lambda_{p}^{L}$ is the associated Laguerre polynomial, with $L$ the azimuthal mode index (topological charge) and $p$ the radial index ( $p=0$ in this work). The variation of the total optical force (sum of the scattering and gradient components) in the axial directions, $F_{z}=Q_{z} n_{1} P_{0} / c_{1}$ where $P_{0}$ is the power in the incident beam, $c_{1}$ the speed of light in vacuum and $n_{1}$ the refractive index of the bubble for an LG beam acting on a $1 \mu \mathrm{m}$ radius air-filled microbubble suspended in water are shown in figure 5(a). Here it can be seen that the net optical force acts to propel the microbubble along the beam axis beyond the beam waist, with a strength that decreases as $L$ increases due to the increasing diameter of the focused LG beam.

2. Find the equilibrium trapping position and the spring constant along the beam axis. As shown in Figure 5a, the force near the focus is repulsive in the axial direction, so the trapping position (if present) must be elsewhere. The equilibrium trapping position must occur along the $z$-axis (due to the symmetry of the radial confinement) and was found by locating the place where the laser force in the z-axis (pushing the bubble downwards) balances the buoyant force acting on the bubble (pushing the bubble upwards) i.e. when

$$
F_{z_{-} \text {total }}=F_{Z}+F_{\text {gravity }}-F_{\text {buoyancy }}=0
$$



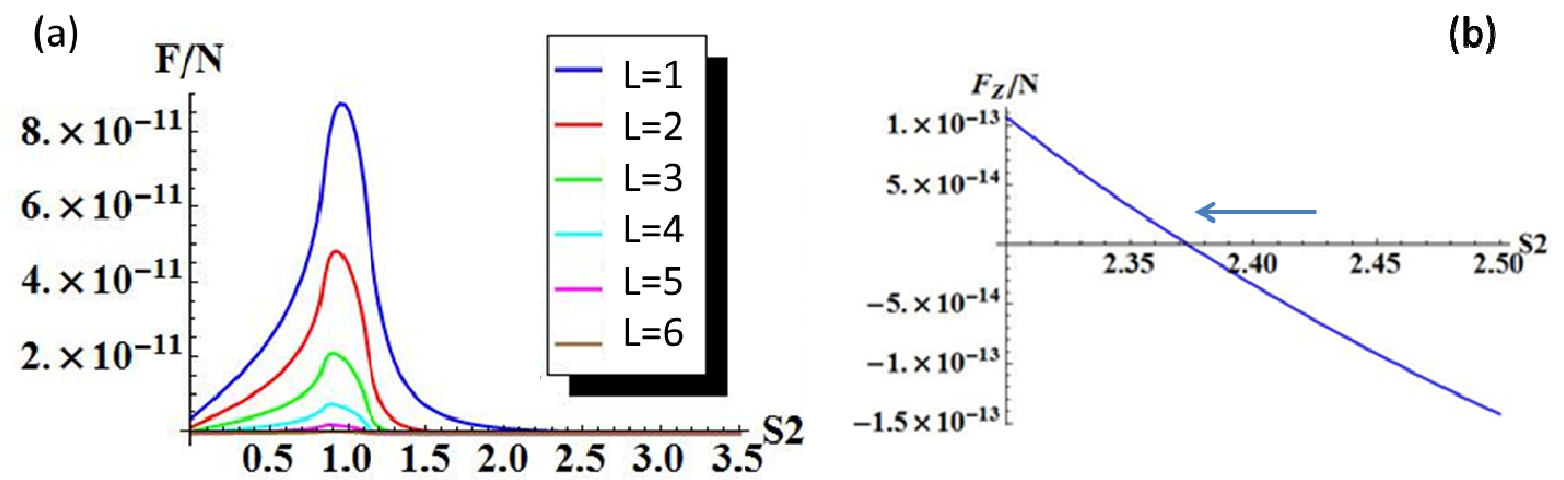

Figure 5. (a) Total optical force (scattering + gradient) along the axis of propagation z-axis (z axis) for a Laguerre Gaussian beam and a low-index microbubble $\left(1 \mu\right.$ m radius, $\left.\mathrm{n}_{1}=1.33, \mathrm{n}_{2}=1.0\right)$; (b) Position of the stable equilibrium for $L=1$, with the arrow indicating the direction of buoyancy.

We deduce, therefore, that the stability of the microbubble in the LG beam trap relies on the contribution of the microbubble buoyancy in a manner similar to the contribution of the solid particle weight in optical levitation experiments using an upwards-propagating beam [17].

3. Select the correct trapping position. The zero for eq. (2) was searched in the region for $\mathrm{S} 2=0-5 a 5 a$, with $a$ being the bubble radius. As shown in Figure 5a, in this region there can be more than one point where equation 2 is satisfied. In a realistic equilibrium position, however, the gradient of the optical force must be negative: the laser would then be pushing the bubble downwards (positive $\mathrm{z}$ direction), counteracted by the buoyancy pushing upwards (negative $\mathrm{z}$ direction) as illustrated in Figure 5(b).

4. Calculate the trap stiffness. Once the trapping position is found, the local gradient of the optical force defined the trap stiffness, respectively for the vertical $k_{z}=-\frac{d F_{z} \text { total }}{d s_{2}}$ and the radial direction $k_{y}=-\frac{d F_{y}}{d s_{1}}$.

\section{RESULTS}

Intuitively, when the diameter of the doughnut beam is much larger than the bubble, the bubble can pass thorough and escape the trap, i.e. there is filtration by size as observed in [10]. Similarly the bubble will also escape when increasing the power: the scattering force would push the bubble downwards until the horizontal force cannot sustain it in the trap against thermal fluctuations. These behaviours were observed in both experiments and modelling results.

\subsection{Simulation results}

The simulation was run according to the experimental values, but covered a larger range of the control parameters: trap characteristics were calculated for a given bubble size as a function of topological charge, $L$, and power, $P_{0}$. In the following calculations, trapped bubbles were assumed to be spherical (bubble diameters, $d_{\text {bubble }}$, of $5 \mu \mathrm{m}, 10 \mu \mathrm{m}, 15 \mu \mathrm{m}$, $20 \mu \mathrm{m}$ ), with $n_{1}=1$ and $n_{2}=1.33$; the objective had a numerical aperture NA $=1.25$ and $l_{\mathrm{t}}=200 \mu \mathrm{m}$.; The trapping laser had a Laguerre Gaussian irradiance profile with topological charge $L=1-10$ and a power $P_{0}=10 \mathrm{~mW}-100 \mathrm{~mW}$. For some combinations of parameters, the method described in section 3 did not identify an equilibrium position, as the buoyancy force was much larger than the maximum optical force in the trap. In these cases, it was assumed that the bubble had escaped the trap: these are indicated as null results in Figures 6-8. The theoretical results indicate that:

- The equilibrium position shifts downwards as the power increases due to the increasing optical force, and decreases as $L$ is increased die to the decreased overlap between the bubble and the high intensity annulus (Figure 6). Larger diameter bubbles have lower equilibrium positions, but are not displaced more than their diameter $\left(d_{\text {bubble }}\right)$.

- Axial trap stiffness $\kappa_{\mathrm{z}}$ has a maximum, whose position in the $\left(P_{0}, L\right)$ space depends on bubble size (Figure 7).

- The radial trap strength decreases with increasing $L$, until bubbles can no longer be trapped, and increases with $P_{0}$ when all the other parameters are kept constant (Figure 8).

- An equilibrium trapping position could be found only when $L<6$; and in this range radial stiffness reach a maximum value of $0.5 \mathrm{pN} / \mu \mathrm{m}$. 

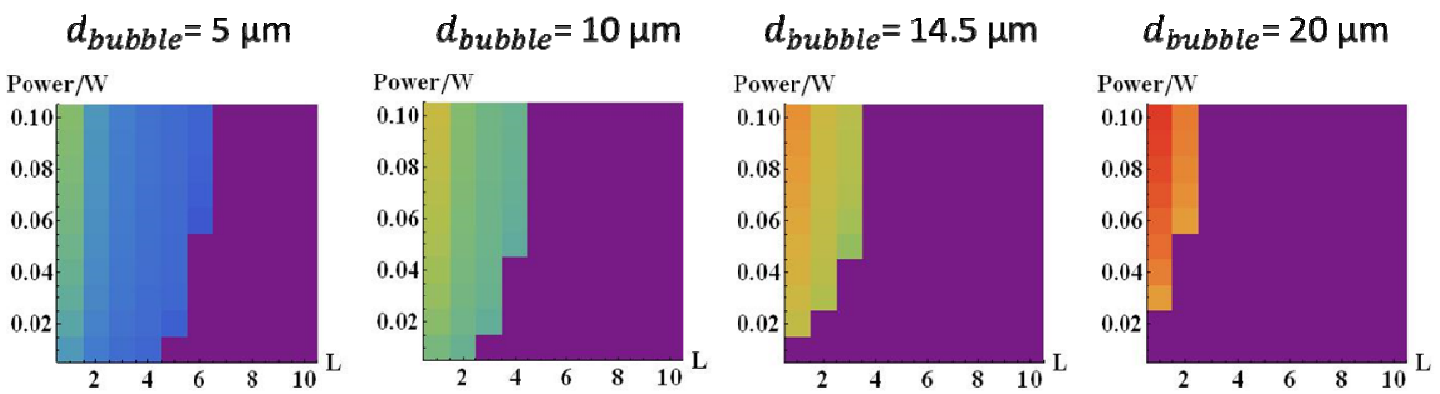

Z Axial trapping position $/ \times 10^{-5} \mathrm{~m}$

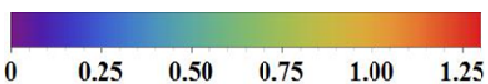

Figure 6: Calculated axial trapping position as a function of power and topological charge $L$.

$$
d_{\text {bubble }}=5 \mu \mathrm{m}
$$

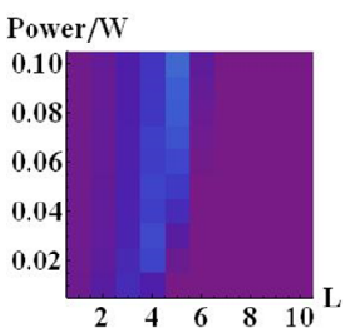

$$
d_{\text {bubble }}=10 \mu \mathrm{m}
$$

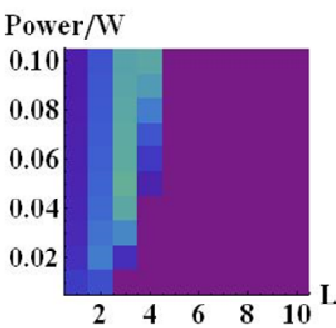

$d_{\text {bubble }}=14.5 \mu \mathrm{m}$

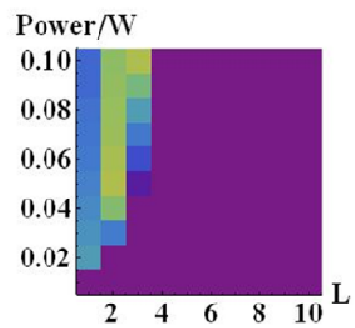

$$
d_{\text {bubble }}=20 \mu \mathrm{m}
$$

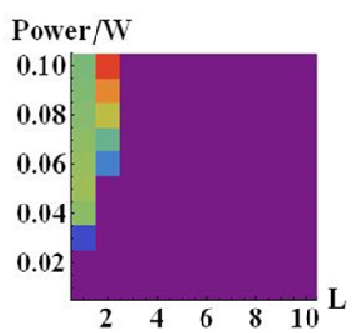

Z Spring constant $/ \times 10^{-4} \mathrm{Nm}^{-1}$

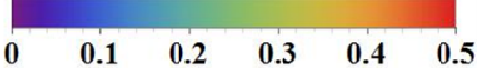

Figure 7: Calculated axial trap stiffness as a function of power and topological charge $L$.
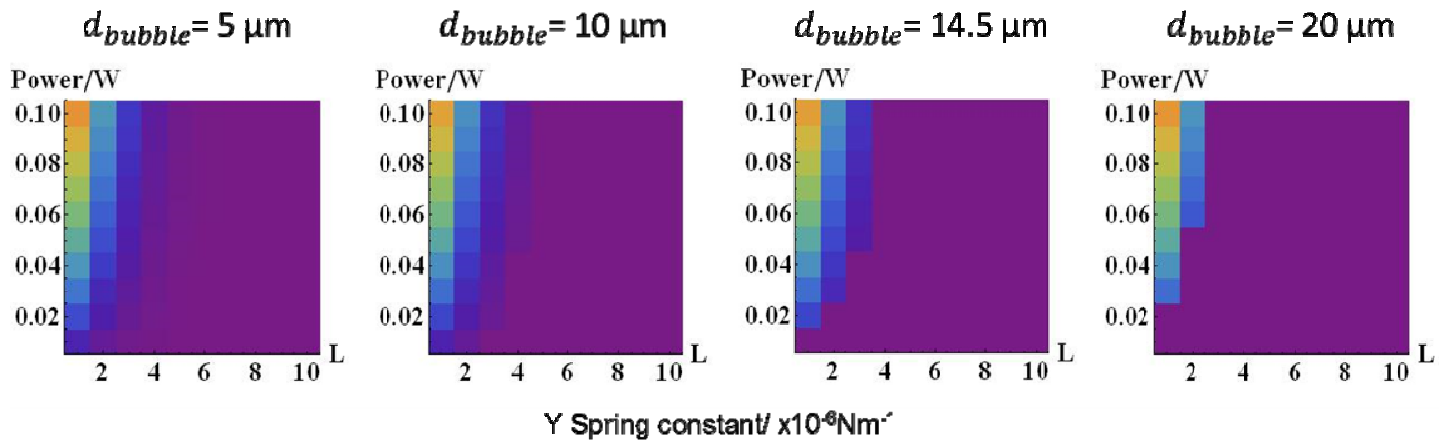

$\begin{array}{lllllll}0 & 0.1 & 0.2 & 0.3 & 0.4 & 0.5\end{array}$

Figure 8: Calculated radial trap stiffness as a function of power and topological charge $L$.

\subsection{Experimental results}

For this study, 50 bubbles of varying diameter (see Figure 2b) were trapped $30 \mu \mathrm{m}$ below the $170 \mu \mathrm{m}$ coverslip and their motion observed for various combinations of parameters: beam topological charge, $L$, beam power, $P_{0}$ and bubble diameter, $d_{\text {bubble }}$. As expected from the modelling, microbubbles were held beyond the beam waist, out of the imaging plane of the camera, so a lensing function was added to the SLM in order to bring the microbubble into focus. 

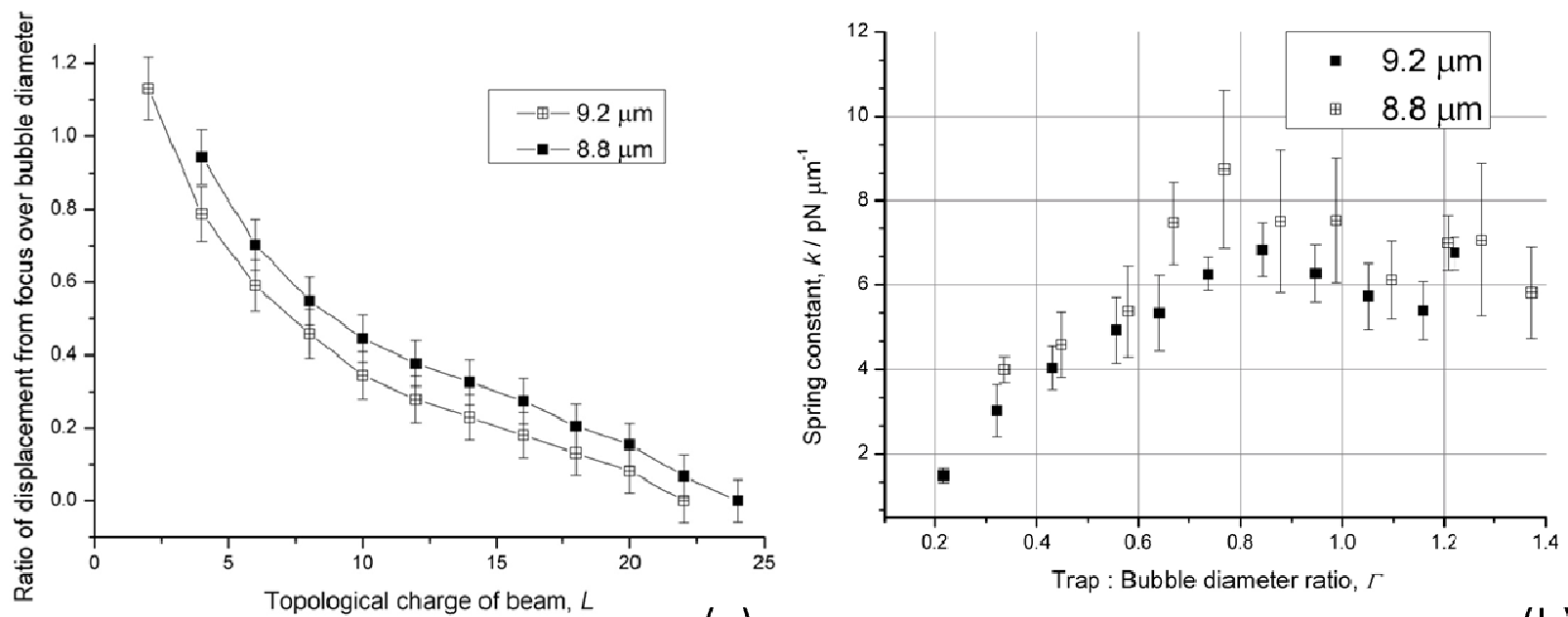

(a)

(b)

Figure 9: Dependence of (a) vertical displacement and (b) radial trap stiffness on $L$ for two bubble diameters (7 repeats each). Laser power was constant at $62 \mathrm{~mW}$.
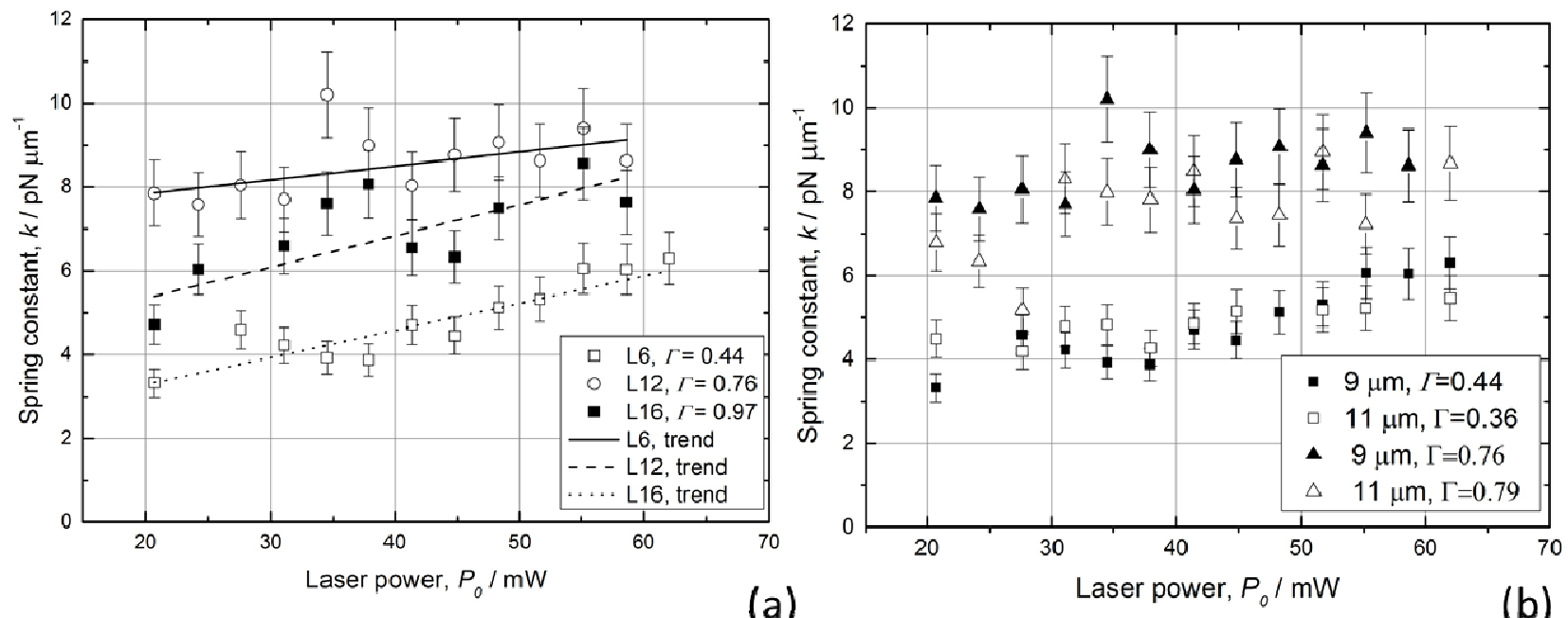

Figure 10 Trap stiffness as a function of laser power for different values of the topological charge $L$ : (a) averaged measurements over three bubbles with diameter $9.0 \pm 0.3 \mu \mathrm{m}$ (linear trend are displayed only as a guidance) and (b) data for two different bubble diameters, grouped by similar bubble-to-trap ratio, $\Gamma$, defined in the text.

Figure 9a reports the vertical displacement of the equilibrium trapping position from the beam waist, relative to the bubble diameter, as a function of the topological charge $L$ for two bubbles, $d_{\text {bubble }}=8.8 \pm 0.2 \mu \mathrm{m}$ and $9.2 \pm 0.2 \mu \mathrm{m}$. Error bars (1 standard deviation) were calculated assuming a resolution of $0.2 \mu \mathrm{m}$ in the measurement of the vertical displacement and propagating the uncertainty of the bubble diameters. The vertical scale is relative to the position at $L=25$, which is expected to be as close to the focus as possible, and the maximum displacement occurs for low value of $L$, where the beam shape is almost Gaussian.

Figure $9 \mathrm{~b}$ presents the measured transverse spring constant, $\kappa$, deduced from the variance of the position fluctuations as a function of trapping beam diameter, $d_{\text {trap }}$, (measured at the beam waist and set by the topological charge, $L$ ) for the same two bubbles. Each curve reports the value averaged over seven different repeats of the same experiment (with the same bubble) and error bars represent one standard deviation. As can be seen in Figure 9b, there is an optimum ratio of $d_{\text {trap }} / d_{\text {bubble }}$ at which the transverse spring constant is maximum. For small trap diameters the overlap between the high intensity part of the beam and the bubble pushes it to an axial equilibrium position beyond the beam waist. The 
divergence of the beam at this position means that the curvature of the optical potential, and hence the measured spring constant are

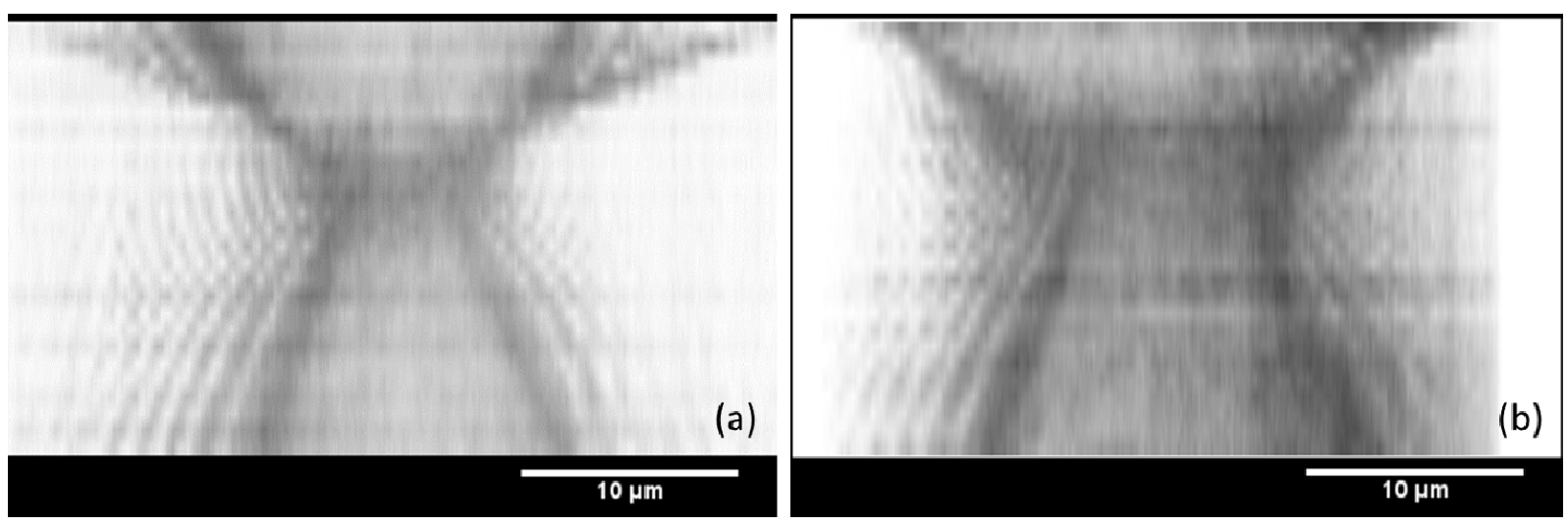

Figure 11. Beam profile (recorded from the reflection of the coverslip at different heights) for (a) $L=12$ and (b) $L=20$.

low. At large beam diameters the spring constant decreases due to the decreasing overlap between the high intensity part of the annular beam and the bubble. These competing processes give rise to the observed maximum in spring constant at a ratio of $\Gamma=d_{\text {trap }} / d_{\text {bubble }}=0.80 \pm 0.05$ for both microbubble diameters .

Finally, Figure 10a shows the dependence of radial trap stiffness on laser power in the range 20-62 $\mathrm{mW}$ (the upper limit is due to the damage threshold of the SLM with the current set-up). Each point in Figure 10a is the average over three bubbles, with diameters in the range $8.7<d_{\text {bubble }}<9.3 \mu \mathrm{m}$ (i.e. the diameter range in Figure 9), for three values of the trap-to-bubble ratio: $\Gamma=0.44(L=6), \Gamma=0.76(L=12)$ and $\Gamma=0.97(L=16)$. Uncertainties were estimated at $10 \%$, from the maximum standard deviation in the values for a single point. The lines, representing a linear trend, show a similar

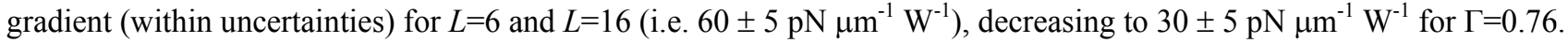
It must be noted, however, that since the three linear trends do not pass from the origin (i.e. stiffness must go to zero at zero power), a non-linear behavior is expected at lower powers.

The presence of a different dependence on power for different values of $\Gamma$ is confirmed in Figure 10b, which reports also the averaged data for three bubbles with a diameter $10.7<d_{\text {bubble }}<11.3 \mu \mathrm{m}$ for $L=6(I=0.36)$ and $\mathrm{L}=16(I=0.79)$. Even in this case, uncertainties were estimated at $10 \%$, from the maximum standard deviation in the values for a single point. Figure $10 \mathrm{~b}$ shows that the trends are similar for similar values of $\Gamma$. Further statistics will be acquired, in future studies, to investigate this dependence in more detail.

\section{DISCUSSION}

While the experiments confirm some of the behaviours predicted by the model (i.e. vertical displacement of the trap as L increases, presence of optimal trapping conditions, monotonic dependence on power), there are some important differences. The most obvious one is that it was possible to trap with much higher values of topological charge (beam diameter) than predicted by the model. One possible reason for the discrepancy is that the model assumes that the radius of the incident LG beam is adjusted to fill the objective aperture. Experimentally this is not always the case, as it would require changing the $4 f$ relay optics system each time the topological charge of the hologram on the SLM is changed. It is possible, therefore, that the filling of the objective back aperture was not optimal for some parameters. Furthermore, the objective used exhibits spherical aberration, which degrades the focusing as shown in Figure 11, and this is not accounted for in the model. The ray optics model reproduced qualitatively the observed experimental behavior of the location of the optical trap being beyond the beam waist, and allowed interpreting the trapping mechanism as a sensitive interplay between optical and buoyancy forces. In this experimental geometry there appears to be an optimum diameter for the trap that maximises the radial stiffness, which was not observed in the time-averaged trap geometry [8].

\section{CONCLUSIONS}

In this work we have examined the effect of the trapping parameters on the radial stiffness of a Laguerre-Gaussian optical tweezers set-up for microbubble manipulation.. We deduce that the action of the trap relies on the combination of 
optical and buoyancy forces acting on the bubble. Varying the control parameters we observed that for a fixed bubble size the displacement from the waist of the axial equilibrium position increased with laser power, but decreased with trapping beam diameter. A maximum in the measured radial trap stiffness when the trap to bubble diameter ratio was $d_{\text {trap }} / d_{\text {bubble }}=0.80 \pm 0.05$ which was not evident in the time-averaged trap geometry studied previously. The transverse spring constant appears to vary linearly with laser power in the region of laser power parameters investigated, although the data is suggestive of nonlinear behavior outside this range. Further studies will look at the axial stiffness to make a quantitative comparison on the efficacy of trapping between the two methods for trapping microbubbles.

\section{ACKNOWLEDGEMENTS}

The authors acknowledge financial support from the NPL Strategic Research Fund (project 114248). ES and CH acknowledge additional support from EPSRC (grant EP/I021795/1). CF acknowledges additional support from University College London's IMPACT scheme.

\section{REFERENCES}

[1] Maragò, O.M., Jones, P. H., Gucciardi, P. G., Volpe, G., Ferrari, A. C. “Optical trapping and manipulation of nanostructures," Nature Nanotechnology, 8, 807-819 (2013).

[2] Dholakia, K., Čižmár, T., "Shaping the future of manipulation," Nature Photon., 5, 335-342 (2011).

[3] Ashkin, A., Dziedzic, J. , Bjorkholm, J., Chu, S.,"Observation of a single-beam gradient optical trap for dielectric particles," Opt. Lett., 11, 288-290 (1986).

[4] Hernot, S., Klibanov, A. L., "Microbubbles in ultrasound-triggered drug and gene delivery," Advanced Drug Delivery Reviews, 60, 1153-1166 (2008).

[5] Blomley, M. J. K., Cooke, J. C., Unger, E. C., Monaghan, M. J., Cosgrove, D. O., "Microbubble contrast agents: a new era in ultrasound," BMJ, 322, 1222 (2001).

[6] Memoli, G., Fury, C.R., Harfield, C. J., Stride, E. P., Jones, P.H., "Optical manipulation of microbubbles in presence of acoustical fields", IEEE International Ultrasonics Symposium, Dresden, DE, http://www.npl.co.uk/upload/pdf/opt-manipulation-microbubbles-memoli.pdf (2012).

[7] Ashkin. A., "Forces of a single-beam gradient laser trap on a dielectric sphere in the ray optics regime," Biophys. J., 61, 569-582 (1992).

[8] Jones, P. H. , Stride, E. , Saffari. N., "Trapping and manipulation of microscopic bubbles with a scanning optical tweezer," Appl. Phys. Letters, 89, 081113 (2006).

[9] Gahagan, K.T., Swartzlander, G.A., "Trapping of low index particles in an optical vortex," J. Opt. Soc. Am. B 15, 524 (1998).

[10] Prentice, P. A., MacDonald, M. P., Frank, T. G., Cuschieri, A., Spalding, G. C., Sibbett, W., Campbell, P. A., Dholakia, K.. "Manipulation and filtration of low index particles with holographic Laguerre-Gaussian optical trap arrays," Optics Express, 12, 593-600 (2004).

[11] Prentice, P. A., Cuschieri, A., Dholakia, K., Prausnitz M., Campbell, P., "Membrane disruption by optically controlled microbubble cavitation," Nature Physics, 1, 107-110 (2005).

[12] Garbin, V., Cojoc, D. , Ferrari, E., Di Fabrizio, E., Overvelde, M. L. J., van der Meer, S. M., de Jong, N., Lohse, D., Versluis, M., "Changes in microbubble dynamics near a boundary revealed by combined optical micromanipulation and high-speed imaging," Appl Phys Lett, 90, 114103 (2007).

[13] Jones, P. H., Maragó, O. M., Stride, E. P. J., "Parametrization of trapping forces on microbubbles in scanning optical tweezers," Journal of Optics A: Pure \& Applied Optics, 9, S278-283 (2007).

[14] Crocker J. C., Grier, D. G., "Methods of digital microscopy for colloidal studies," J. Coil. Interf. Sci., 179, 298310 (1996).

[15] http://www.npl.co.uk/science-technology/dimensional/services/npl-reference-stage-graticules

[16] Jones. P. H., "Optical Tweezers," [Encyclopedia of Optical Engineering], 172, PP 1-16, DOI 10.1081/E-EOE120047157

[17] Ashkin, A., Dziedzic, J.M., “Optical levitation by radiation pressure,” Appl. Phys. Lett., 19 (8), 283-285 (1971). 